\title{
Data Content Weighing for Subjective versus Objective Picture Quality Assessment of Natural Pictures
}

\author{
Suresha D \\ Research Scholar, Visvesvaraya Technological University, Belagavi, 590018, India. \\ Email: sureshasss@gmail.com \\ H N Prakash \\ Professor and Head, Department of Computer Science and Engineering, Rajeev Institute of Technology, Hassan, \\ 573201, India. \\ Email: prakash_hn@yahoo.com
}

\begin{abstract}
Estimating the visual quality of picture is a real challenge for various picture and video frame applications. The aim is to evaluate the quality of picture automatically in both subjective (human visual frame work) and objectively. The quality of picture is evaluated by comparing precision and closeness of a picture with reference or error free picture. The quality estimation can be done to achieve consistency in desired quality of picture with help of modeling remarkable physiological, psycho visual components framework and picture fidelity measure methods. In this article, the picture quality is evaluated by analyzing loss of picture information of the distortion system using differing noise models and examine the relationship between picture data, visual quality and error metric. The quality of picture \& video frame assessment is really important that, every human can judge the visual quality of natural picture. The subjective quality of picture is assessed by using structural similarity metric, objective quality of picture is computed by root means squared error, mean squared error and peak signal to noise ratio and data content in picture is weighted through entropy.
\end{abstract}

Index Terms-Gaussian, Local Variance, Poisson, Salt and Pepper, Speckle, Structural SIMilarity, Mean Squared Error, Root Mean Squared Error, Peak Signal to Noise Ratio, Entropy.

\section{INTRODUCTION}

In recent years, digital picture (DP) / video frame (VF) handling is a noteworthy field of examination in the region of computer science and communication engineering. It is subsequently increased in developing the interest of picture and video administrations. Be that as it may, advanced picture handling experience the ill effects of inherent noise from their phase of capturing [1], compressing[2], encoding[3], communication[4], decoding and processing stages[5]. This noise appearance will change the normal arrangement of original data in DP/VF signal.

Investigation of picture quality estimation is essentially done with various noise models. The digitized pictures are broadly utilized in the area of medicine for diagnosis of medical pictures like calcification in mammographic pictures[6], film/ video creation[7][8] and photography of natural pictures for psycho visual components of the human visual framework(HVF)[9], remote detecting satellites for recognition of components at far separation[10], military target distinguishing proof and their examination[11], and assembling mechanization and control of segments[12]. It is essential for the DP processing to distinguish and evaluate the quality debasements with an end goal to keep up the required quality of service. This offers the computation of ascend longing of exact \& productive perceptual picture quality assessment (PQA), that can evaluate the subjective nature of the picture content under different sorts of distortions.

Hence the proposed work begin with noise models and the role of noise in picture deformation where noise is arbitrary signal utilized to decimate the part of picture data and picture distortion is pleasance issues in picture processing. Picture distorted because of different sorts[13] of noise, for example, Gaussian(Ga)[14][1], Local Variance $(\mathrm{Lv}), \quad$ Poisson(Po)[15], Salt and Pepper $(\mathrm{S} \& \mathrm{P})[16]$ and Speckle (Sp)[17] noise in case of DP. These noises in the picture might be originated from the improper catching gadgets [1] like cameras, misaligned lenses, improper focal length and dissipating. Then we attempt to co relate mutilated picture with "reference" or " flawless " picture subjectively by utilizing HVF very adapted for extracting structural data from a scene which is measured through structural similarity (SSIM)[18][9]. Then it attempted to co-relate objectively by using mean squared error (MSE)[9], root mean squared error (RMSE)[9] and peak signal to noise ratio(PSNR)[9]. Later the details of picture are weighed through statistical measure of haphazardness that can be utilized to portray the composition of the picture by means of entropy(EnT)[19][20]. 
The rest of this paper is organized as follows: The section 2 discusses the detailed literature survey, section 3 illuminates proposed architecture with different noise function and comparison of picture contents. The observations and performance analysis are discussed in section 4 with SSIM, Entropy, MSE, RMSE and PSNR finally we have summarized the article in the section 5.

\section{LITERATURE REVIEW}

Examination of picture quality is fundamental in several areas of digital picture processing. The picture quality calculations are broadly classified into two categories, first category uses a reference or clear picture for comparison with generated picture and second category calculates data content in generated picture.

The superiority of generated picture in first category of PQA is decided by accuracy or differences generated by picture with reference picture. These computation metrics on quality of picture are divided into objective PQA computation and subjective PQA computation metric. The objective PQA computation will not consider HVF while designing metrics, instead they use pixel by pixel differences, square of their differences, absolute differences between pixels or maximum signal to noise ratio. The objective of these calculation is to have very low value for difference i.e. zero for perfect generated image and very high value for maximum signal to noise ratio i.e. infinity for perfect generated picture. The state of art calculations such as MSE, RMSE, mean absolute error (MAE) and PSNR generally used in measuring objective PQA. These category of calculation fit well for signal processing domain, but needs to be improved for picture processing classes where human can judge the visual quality of picture. The subjective PQA depends on human visual quality metric, which is hard to put into calculation[8][10] but these quality metric has been tried with structural similarity metric[18]. The subjective PQA use SSIM calculation and aim to achieve 1.0 for perfect construction image.

The quality of generated picture in second category of PQA is determined by equalizing data content in generated picture with reference picture. The calculations on these category measure statistical arbitrariness in picture surface which is calculated by EnT of a picture.

Many researchers have used the different quality metrics to prove their performance in achieving the high quality of picture. The author of [2] have discussed compression technique on DCT based picture compression in which PSNR is used to measure the performance of generated pictures over JPEG and JPEG2000. In designing mathematical model[3] for the noise in binary coding of multiplexed signals in imaging system signal to noise ratios are used in deciding performance. In designing post processing of JPEG-2000 low bit rate compression technique have been discussed in[5] to eliminate coding artifacts in which PSNR is used as a standard for deciding quality. The author of [6] have discussed the pre-processing of mammogram pictures for breast cancer detection in which picture quality is decided through MSE, PSNR, structural content(SC) and normalized absolute error(NAE). The quality of satellite pictures after their increase in resolution[10] are quantitatively compared through PSNR and picture visual content for demonstrating their superiority. The identification of enemy targets in synthetic aperture radar pictures are discussed in [11] which elaborates with more accuracy with binary image matching method for estimation of the clear target. The author of [19] increased the rate of identification of picture through unique identifier generation, which is the fusion of entropy with features of picture. For comparing the quality of super resolution picture with reference picture RMSE, MSE, PSNR are used in [21] and MSE,PSNR, EnT and Q-INDEX are used in [22]. The picture resolution up-sampling technique in [23] use PSNR with visual picture to decide the quality of generated up sampled picture.

The many researchers have used objective PQA calculation in presenting their quality results. But evaluating the quality of picture automatically in both subjective and objectively must be done to achieve the high quality of picture. The quality of picture should be assessed by objective PQA, subjective PQA and data content weighing. This paper focus on using various noise models on natural pictures and its implication on objective, subjective quality measures along with their data content.

\section{PROPOSED ARCHITECTURE OF EXPERIMENTATION}

The proposed architectural design of data content weighing for subjective versus objective PQA is shown in Fig. 1. The experimentation setup receives reference natural picture (NaP) shown in Fig. 7 as an input to noise function (NoF) and results in noisy picture (NoP) as shown in (1). The data details of $\mathrm{NaP}$ and NoP are weighed by statistical measure of irregularity EnT, the HVF of NaP and NoP is measured by SSIM and objective quality by RMSE, MSE and PSNR.

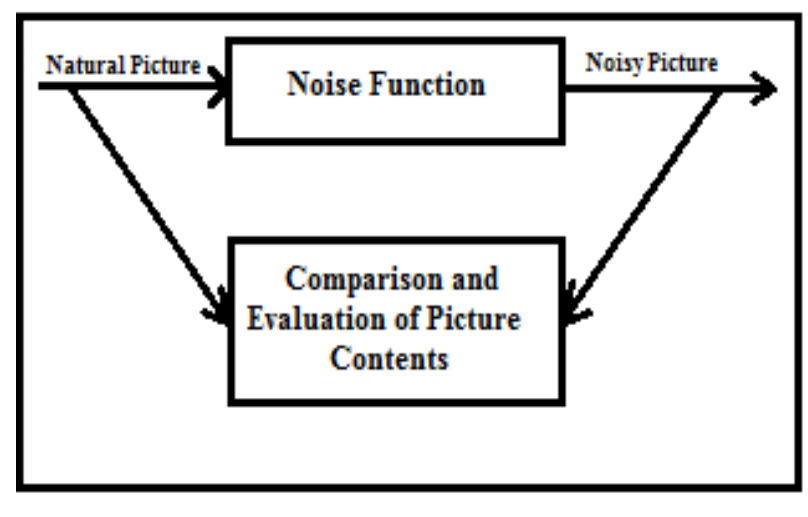

Fig.1. Proposed design of experimentation

$\mathrm{NaP}$ is subjected to NoF results in NoP as depicted in (1).

$$
N_{o} P=N_{o} F\left(N_{a} P\right)
$$


The NoF considered for experimentation are $G a, L v$, $P o, S \& P$ and $S p$ noise types discussed in section B.

\section{A. Notations Used}

The notations used in this article are described in table 1 as follows.

Table 1. Notations used in the article

\begin{tabular}{|c|l|}
\hline Notation & \multicolumn{1}{|c|}{ Explanation } \\
\hline NaP & Natural Picture \\
\hline NoP & Noisy Picture \\
\hline HVF & Human Visual Framework \\
\hline PQA & Picture Quality Assessment \\
\hline Ga & Gaussian \\
\hline Lv & Local Variance \\
\hline Po & Poisson \\
\hline S\&P & Salt and Pepper \\
\hline Sp & Speckle \\
\hline SSIM & Structural Similarity \\
\hline MSE & Mean Squared Error \\
\hline RMSE & Root Mean Squared Error \\
\hline PSNR & Peak Signal to Noise Ratio \\
\hline EnT & Entropy \\
\hline NAE & Normalized Absolute Error \\
\hline SC & Structural Content \\
\hline MAE & Mean Absolute Error \\
\hline
\end{tabular}

\section{B. Noise Function}

Noise function produces and describes undesirable data in digitized pictures, for example artifacts, farfetched edges, concealed lines, corners and obscured objects by disturbing background details. To minimize these undesirable impacts, prior learning of different noise function is fundamental for effective handling of NoP. Advanced noises might emerge from different sources through different sensors. The noise from sensors are modelled through probability density function (PDF)[14] or histogram. Hence the different noise models which are applied on $\mathrm{NaP}$ for experimental result as follows.

\section{Gaussian(Ga) Noise:}

It is a white electronic distortion during signal amplification or recognition. $G a$ is brought by normal sources like vibration of particles and radiation of objects. $G a$ aggravates the pixel values in computerized pictures. That is why $G a$ basically designed and characterized by normalizing histogram with pixel value.

$G a$ shown in (2)[14] includes mean $\mu$ and variance $V a$ of $\mathrm{NaP}$. For experimentation $0 \mu$ noise with 0.02 and 0.03 times of $V a$ is considered and its comparison is tabulated in table $3 \& 4$ respectively.

$$
G_{a}=\frac{1}{\sqrt{2 \pi s_{d}}} e^{-\left(N_{a} P-\mu\right)^{2}} / 2 V_{a}
$$

\section{Local variance $(L v)$ Noise:}

It is a special $G a$ noise[13] to a NaP. $L v$ of the noise function is the function of normalized image intensity value of $\mathrm{NaP}$.

\section{Poisson (Po) Noise:}

The presence of this noise in picture is because of statistical property[15] in electromagnetic waves. For example, visible light, $x$ ray and gamma ray. The gamma and $\mathrm{x}$ rays source radiates the number of photons. These radiated photons are measured in unit time. The radiated rays are infused into patient body from their generation, in medical imaging frameworks. The patient body make irregular change to photons in the radiated rays. Results accumulated picture will have spatial with transient irregularity. This noise is additionally termed as quantum, photon or shot noise. These noise are created from picture as opposed to add fake noise to the picture. Probability distribution function(Podf) by poisson distribution is given by (3).

$$
P_{o} d f(\partial=n)=\frac{e^{-\Lambda t}(\Lambda t)^{n}}{n !} k=0,1,2, \ldots
$$

In (3) $\partial$ is a positive parameter measured as a count of photons given by sensors in time interval $n$. $\alpha$ is the expecting count of photons in time interval $n$. $\partial$ is directly related to the incident location irradiance. The Podf with $\kappa t$ refers to the expecting incident photon measure.

\section{Salt and pepper $(S \& P)$ Noise:}

This is termed as information drop noise[16] due to its change in original values. However the picture is not completely corrupted by $\mathrm{S} \& \mathrm{P}$ noise rather than some pixel amplitudes are changed in the picture. In spite, the fact that noisy picture, there is a potential outcomes of a few unchanged neighbours. This category of noise is found during information transfer. Picture pixel amplitudes are supplanted by changed pixel amplitudes either most least or highest pixel which i.e., 0 or 255 if count of bits are 8 for transfer. This noise is embedded with dead pixels either dull or intense. S\&P adds noise to the NaP, where probability 1 is the likelihood of salt noise and probability 2 is the likelihood of pepper noise. The total probability will be sum of probability 1 and probability 2 will be normalized with total count of the pixels. This influences the product of probability and number of components of $\mathrm{NaP}$ pixels as shown by (4).

$$
\mathrm{S} \& P=\left\{\begin{array}{lr}
255 & \text { probability } 1 \\
0 & \text { probability } 2 \\
N a P & 1-\text { Total probability }
\end{array}\right.
$$




\section{Speckle (Sp) Noise:}

This is a multiplicative noise[17] to the picture $\mathrm{NaP}$. The $S p$ is observed in imaging framework like laser, radar and acoustics and so forth. Speckle noise can exist comparative in an picture as $G a$ noise. Its likelihood follows gamma distribution, Noise function of $\mathrm{Sp}$ is portrayed by (5).

$$
S p=\eta_{m u l} * N a P+\eta_{\text {add }}
$$

Where $N a P$ is Natural Picture and $\eta$ will be uniformly distributed random noise where $\eta_{m u l}$ is multiplicative and $\eta_{\text {add }}$ is additive constant. For experimentation, 0 mean noise with 0.02 and 0.03 times of variance is considered.

\section{Measurement metric of Picture}

PQA tries to measure a visual quality of picture, a measure of distortion in a given picture. These distortions are inescapable part of any digital picture handling phase of acquiring, compressing, encoding, transmission, deciphering and processing stages.

\section{Objective PQA}

The objective PQA measures a contrast between two pictures and the outcome can be comprehended with level of errors between pictures. Their measure is public, effectively computable by legitimate distance metric and gives consistent understanding of picture similarity. As all result of this MSE, RMSE and PSNR turned into a convention[21][22][23] in objective PQA.

The mathematical formulation for MSE, RMSE and PSNR [9] are represented by (6), (7) and (8) respectively.

$$
M S E=\frac{1}{S_{z}} \sum_{z=1}^{3} \sum_{y=0}^{n-1} \sum_{x=0}^{m-1}(N a P(x, y, z)-N o P(x, y, z))^{2}
$$

$$
\begin{gathered}
\text { Where } S_{z}=m X n X 3 \\
m: \text { number of rows } \\
n: \text { number of columns } \\
\text { RMSE }=\sqrt{\mathrm{MSE}} \\
\text { PSNR }=10 \log _{10} \frac{M_{x}^{2}}{M S E}
\end{gathered}
$$

Mx: Maximum value of gray level of pixel(255)

Based on various experimental observation, the objective PQA doesn't give much information to human perceived quality of picture. This prompted endeavours to make measures, whose performance would be firmly identified with the human perception of visual appearance.

\section{Modelling Human Visual Framework}

The contrast between pictures does not quantify the distortion. It attempts to characterize the model of human visual framework (HVF)[18][9]. The main correct technique for assessing the human perceived visual quality of pictures is the assessment by people.
Unfortunately, such method is not usable in progressive applications. Hence, there is a requirement for an automated technique, that would predict the humanperceived visual quality as close as expected under the above circumstances.

In this framework all parts of HVF were absolutely modeled and exact prediction of subjective picture visual quality of picture which would be most likely accomplished. Exact modeling of HVF is difficult to achieve, because HVF is very complicated framework with a lot of nonlinearities. SSIM is a measure in view of the assumption that HVF is adjusted to extract structural information from the field of perspective. With these, the change of basic structural information amongst distorted and original picture could be a good estimation of observed picture distortion. SSIM works well if it utilized locally and contrasts the pixel-to-pixel like objective PQA. SSIM is scientifically figured according to the (9) is collected by a comparison of luminance, contrast and structure of $\mathrm{NaP}$ and NoP in grey level. First, the luminance is compared by a function $l(N a P, N o P)$ of mean intensities $\mu_{\mathrm{NaP}}$ and $\mu_{\mathrm{NoP}}$, Contrast comparison is a function $\mathrm{c}(\mathrm{NaP}, \mathrm{NoP})$ of standard deviations $\mathrm{S}_{\mathrm{d}_{\mathrm{NaP}}}, \mathrm{S}_{\mathrm{d}_{\mathrm{NoP}}}$ and variances $\mathrm{V}_{\mathrm{a}_{\mathrm{NaP}}}, \mathrm{V}_{\mathrm{a}_{\mathrm{NOP}}}$, Finally, structure comparison is a function $\mathrm{s}(\mathrm{NaP}, \mathrm{NoP})$ of correlation between the NaP and $\mathrm{NoP}$ of standard deviations $S_{\mathrm{d}_{\mathrm{NaP}}}, \mathrm{S}_{\mathrm{d}_{\mathrm{NoP}}}$ with $\mathrm{S}_{\mathrm{d}_{\mathrm{NaP}, \mathrm{NOP}}}$.

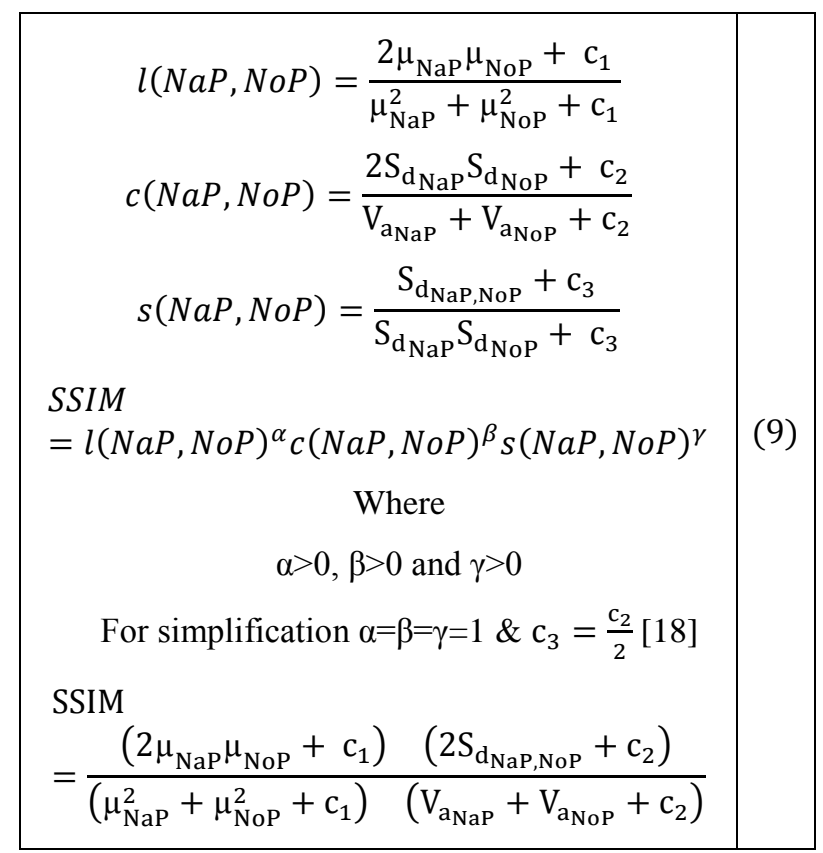


$\mu_{N a P}:$ Mean of $\mathrm{NaP}$

$\mu_{N o P}:$ Mean of NoP

$V_{a_{N a P}}:$ Variance of $\mathrm{NaP}$

$V_{a_{N o P}}:$ Variance of NoP

$\mathrm{S}_{\mathrm{d}_{\mathrm{NaP}}}:$ Standard deviation of $\mathrm{NaP}$

$\mathrm{S}_{\mathrm{d}_{\mathrm{NoP}}}:$ Standard deviation of NoP

$S_{d_{N a P, N o P}}:$ Standard deviation of NaP and NoP

$c_{1}, c_{2}$ and $c_{3}$ : The stabilizing variables for division with weak denominator

\section{Evaluation of Picture Contents}

Entropy(EnT) is a statistical calculation for arbitrariness[19][20] in characterizing input picture surface. EnT is characterized by (10) as

$$
\operatorname{EnT}=-\operatorname{sum}(p \cdot * \log 2(p))
$$

where $\mathrm{p}$ contains the histogram counts

\section{EXPERIMENTAL OBSERVATIONS AND DISCUSSION}

The NaP pictures for experimentation shown in Fig.7. The experimentation is led on dataset[24] for estimation of PQA. Table 2 is the tabulation of EnT of the input $\mathrm{NaP}$ pictures shown in Fig. 7 calculated as per (10).

Table 2. Statistics of EnT for $\mathrm{NaP}$

\begin{tabular}{|c|c|}
\hline NaP & EnT \\
\hline 1.tiff & 6.8981 \\
\hline 2.tiff & 6.2945 \\
\hline 3.tiff & 7.7502 \\
\hline 4.tiff & 7.4858 \\
\hline
\end{tabular}

The input NaP pictures shown in Fig. 7 are subjected to Ga noise as per (2) with 0 mean, 0.02 variance, and resultant noisy pictures displayed in Fig. 8. Ga noise with 0 mean, 0.03 variance displayed in Fig. 9. The pictures of Fig. 7 are contrasted with noisy pictures Fig. 8 and Fig. 9 as per (6)-(10) and tabulated in table $3 \& 4$ respectively.

Table 3. Statistics of Ga noise with 0 mean and 0.02 times of variance

\begin{tabular}{|c|c|c|c|c|c|}
\hline Picture & SSIM & MSE & RMSE & PSNR & EnT \\
\hline 1.tiff & 0.8923 & 17.1797 & 4.1448 & 35.7806 & 7.1934 \\
\hline 2.tiff & 0.9299 & 9.0492 & 3.0082 & 38.5647 & 6.4818 \\
\hline 3.tiff & 0.8148 & 31.3242 & 5.5968 & 33.1720 & 7.8022 \\
\hline 4.tiff & 0.8605 & 27.9207 & 5.2840 & 33.6715 & 7.5766 \\
\hline
\end{tabular}

Table 4. Statistics of Ga noise with 0 mean and 0.03 times of variance

\begin{tabular}{|c|c|c|c|c|c|}
\hline Picture & SSIM & MSE & RMSE & PSNR & EnT \\
\hline 1.tiff & 0.8521 & 24.5115 & 4.9509 & 34.2371 & 7.1885 \\
\hline 2.tiff & 0.9029 & 13.0878 & 3.6177 & 36.9622 & 6.4672 \\
\hline 3.tiff & 0.7544 & 41.7774 & 6.4635 & 31.9214 & 7.8135 \\
\hline 4.tiff & 0.8113 & 37.9993 & 6.1644 & 32.3330 & 7.5989 \\
\hline
\end{tabular}

The analysis of table $3 \& 4$ on SSIM and PSNR reveals that even though the subjective SSIM is high but its objective PQA PSNR is low for picture 4.tiff of table 3 when compared to picture 1.tiff of table 4 of subjective SSIM and objective PSNR.

The NaP pictures shown in Fig. 7 are subjected to $\mathrm{Lv}$ noise with 0.01 random noise, resultant noisy pictures are displayed in Fig. 10. The pictures of Fig. 7 are contrasted with noisy pictures Fig. 10 as per (6)-(10) and tabulated in table 5.

Table 5. Statistics of Lv noise with 0.01 random noise

\begin{tabular}{|c|c|c|c|c|c|}
\hline Picture & SSIM & MSE & RMSE & PSNR & EnT \\
\hline 1.tiff & 0.5672 & 56.9777 & 7.5484 & 30.5738 & 7.1406 \\
\hline 2.tiff & 0.5732 & 47.5187 & 6.8934 & 31.3622 & 6.3194 \\
\hline 3.tiff & 0.5450 & 64.1639 & 8.0102 & 30.0579 & 7.8379 \\
\hline 4.tiff & 0.6108 & 64.1656 & 8.0103 & 30.0578 & 7.6949 \\
\hline
\end{tabular}

The analysis table 5 on SSIM and PSNR reveals that even though the subjective SSIM is high but its objective PSNR is low for picture 4.tiff when compared to picture 3.tiff of subjective SSIM and objective PSNR.

The NaP pictures shown in Fig. 7 are subjected to Po noise as per (3) resultant noisy pictures are displayed in Fig. 11. The pictures of Fig. 7 are contrasted with noisy pictures Fig. 11 as per (6)-(10) and tabulated in table 6.

Table 6. Statistics of Po noise

\begin{tabular}{|c|c|c|c|c|c|}
\hline Picture & SSIM & MSE & RMSE & PSNR & EnT \\
\hline 1.tiff & 0.8686 & 24.1362 & 4.9129 & 34.3041 & 7.2207 \\
\hline 2.tiff & 0.9316 & 14.6803 & 3.8315 & 36.4634 & 6.5031 \\
\hline 3.tiff & 0.7400 & 44.5790 & 6.6768 & 31.6395 & 7.8017 \\
\hline 4.tiff & 0.7158 & 50.3413 & 7.0952 & 31.1116 & 7.6605 \\
\hline
\end{tabular}

The analysis of table 6 on SSIM and PSNR reveals that the subjective SSIM is in-line with objective PSNR.

The NaP pictures shown in Fig. 7 are subjected to S\&P noise as per (4) with 0.01 density resultant noisy pictures are displayed in Fig. 12. The pictures of Fig. 7 are contrasted with noisy pictures Fig. 12 as per (6)-(10) and tabulated in table 7 .

Table 7. Statistics of S\&P noise with 0.01 density

\begin{tabular}{|c|c|c|c|c|c|}
\hline Picture & SSIM & MSE & RMSE & PSNR & EnT \\
\hline 1.tiff & 0.7789 & 1.1612 & 1.0776 & 47.4815 & 6.9200 \\
\hline 2.tiff & 0.7796 & 0.9008 & 0.9491 & 48.5847 & 6.3224 \\
\hline 3.tiff & 0.7743 & 1.2777 & 1.1304 & 47.0665 & 7.7626 \\
\hline 4.tiff & 0.8127 & 1.2608 & 1.1228 & 47.1244 & 7.4956 \\
\hline
\end{tabular}


The analysis of table 7 on SSIM and PSNR reveals that even though the subjective SSIM is high but its objective PSNR is low for picture 4.tiff when compared to picture 1.tiff of subjective SSIM and objective PSNR.

The input NaP pictures shown in Fig. 7 are subjected to Sp noise as per (5) with 0.02 density resultant noisy pictures are displayed in Fig. 13 and $\mathrm{Sp}$ noise with 0.03 density displayed in Fig. 14. The pictures of Fig. 7 are contrasted with noisy pictures Fig. 13 and Fig. 14 as per (6)-(10) and tabulated in table $8 \& 9$ respectively.

Table 8. Statistics of Sp noise with 0.02 density

\begin{tabular}{|c|c|c|c|c|c|}
\hline Picture & SSIM & MSE & RMSE & PSNR & EnT \\
\hline 1.tiff & 0.8373 & 33.9324 & 5.8252 & 32.8247 & 7.2059 \\
\hline 2.tiff & 0.9398 & 16.7352 & 4.0909 & 35.8945 & 6.4381 \\
\hline 3.tiff & 0.5744 & 74.6813 & 8.6418 & 29.3987 & 7.7500 \\
\hline 4.tiff & 0.5153 & 84.6488 & 9.2005 & 28.8546 & 7.7567 \\
\hline
\end{tabular}

Table 9. Statistics of Sp noise with 0.03 density

\begin{tabular}{|c|c|c|c|c|c|}
\hline Picture & SSIM & MSE & RMSE & PSNR & EnT \\
\hline 1.tiff & 0.7848 & 41.7593 & 6.4621 & 31.9233 & 7.2142 \\
\hline 2.tiff & 0.9174 & 21.6677 & 4.6549 & 34.7727 & 6.4375 \\
\hline 3.tiff & 0.5073 & 82.9405 & 9.1072 & 28.9431 & 7.7192 \\
\hline 4.tiff & 0.4558 & 91.4773 & 9.5644 & 28.5177 & 7.7286 \\
\hline
\end{tabular}

The analysis table $8 \& 9$ on SSIM and PSNR reveals that even though the subjective SSIM is high but its objective PQA PSNR is low for picture 4.tiff of table 8 when compared to picture 3.tiff of table 9 of subjective SSIM and objective PSNR.

Each parameter comparisons from all the tables are represented in-terms of graph in which the result of parameter of one tables with same parameter of other table as shown in the Fig 2 - 6.

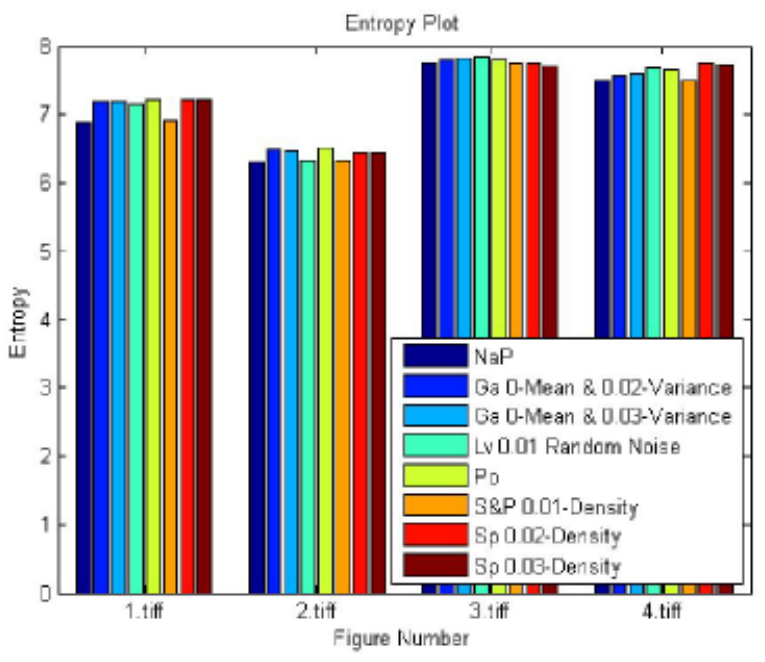

Fig.2. Entropy comparison plot of table 2 - 9

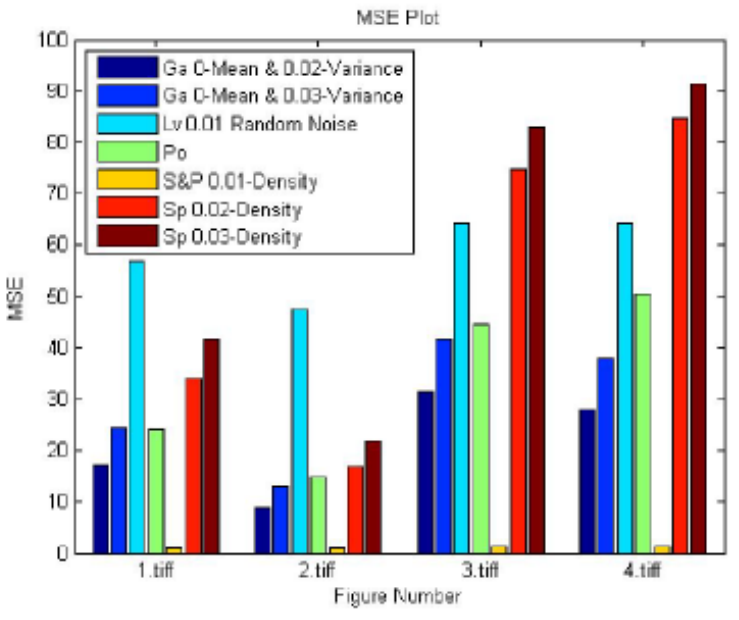

Fig.3. MSE comparison plot of table 3 - 9

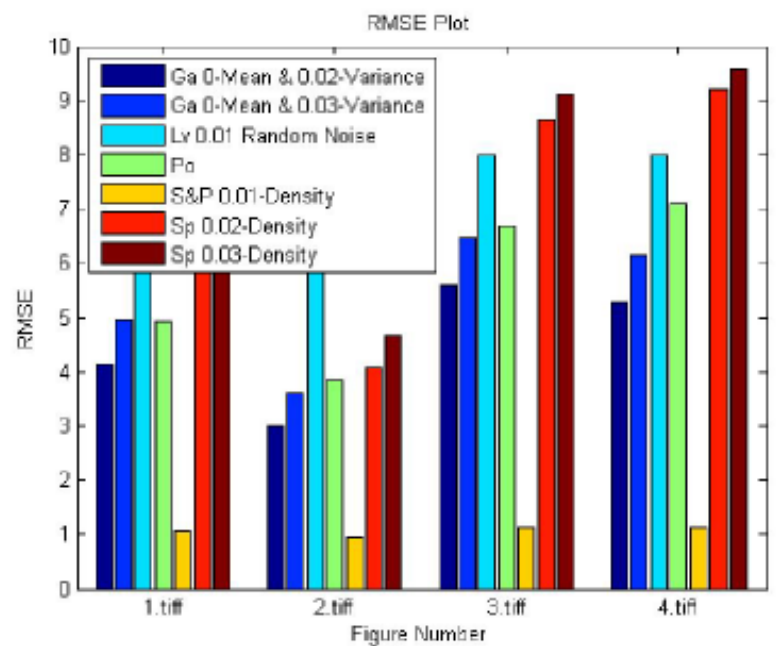

Fig.4. RMSE comparison plot of table 3 - 9

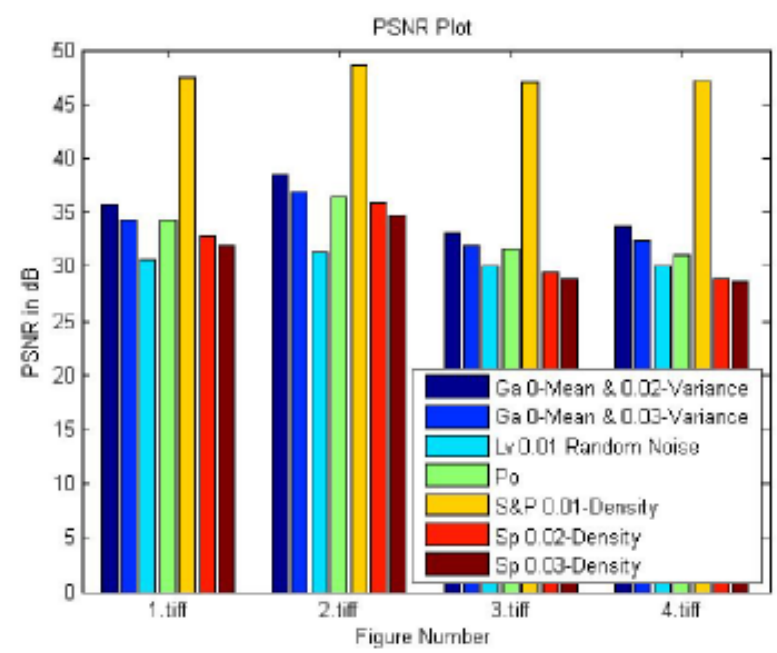

Fig.5. PSNR comparison plot of table 3 - 9 


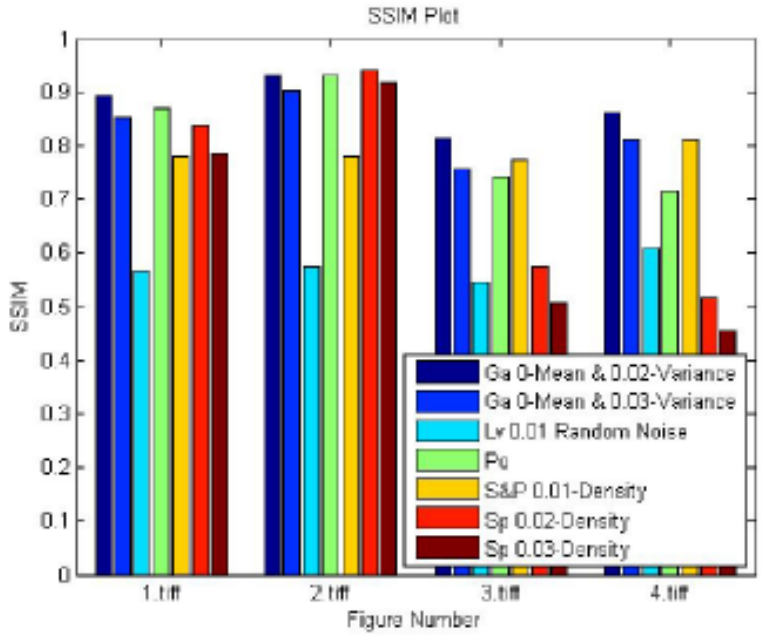

Fig.6. SSIM comparison plot of table 3 - 9

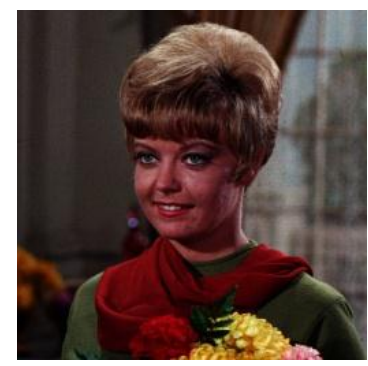

1.tiff

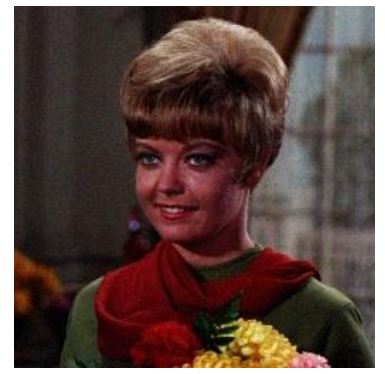

1.tiff

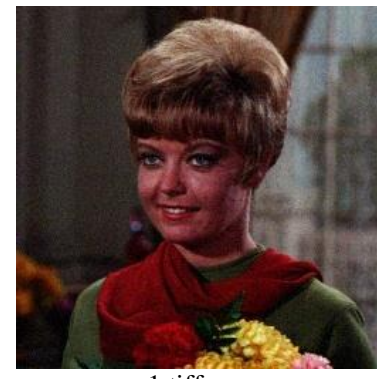

1.tiff

2.tiff

2.tiff
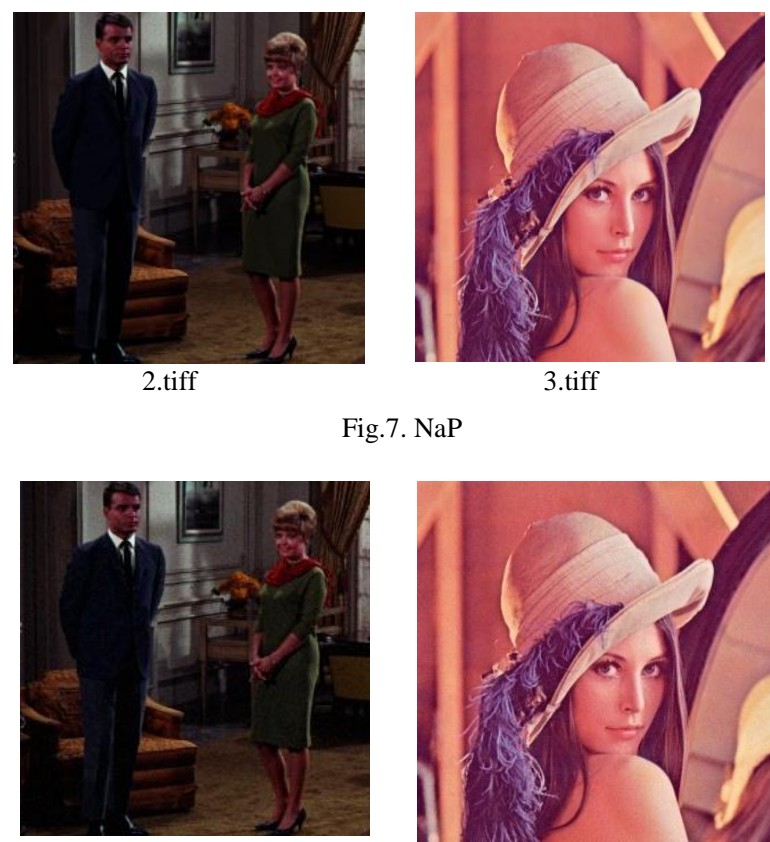

3.tiff

Fig.7. NaP

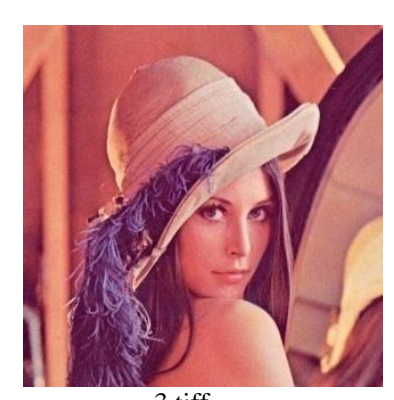

3.tiff

Fig.8. Noisy pictures of Ga noise with 0 mean and 0.02 times of variance

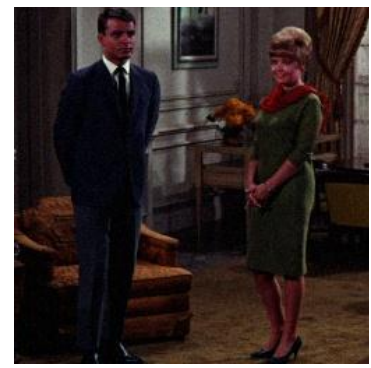

2.tiff

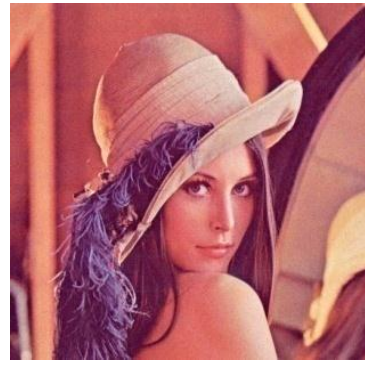

3.tiff

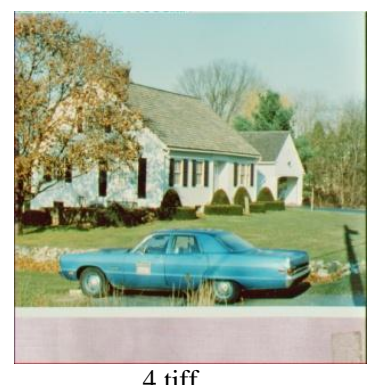

4.tiff

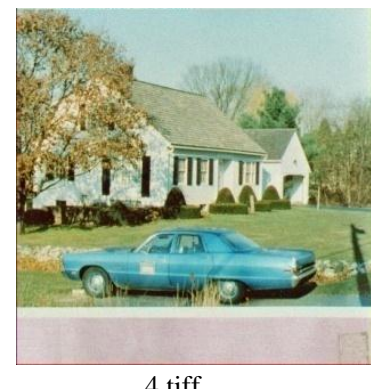

4.tiff

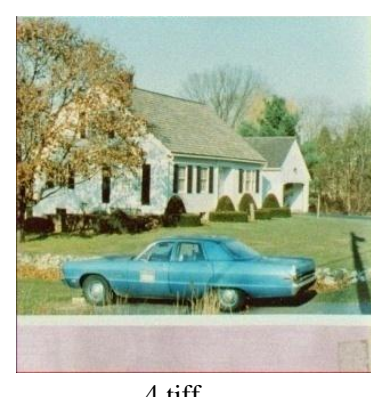

Fig.9. Noisy pictures of Ga noise with 0 mean and 0.03 times of variance 


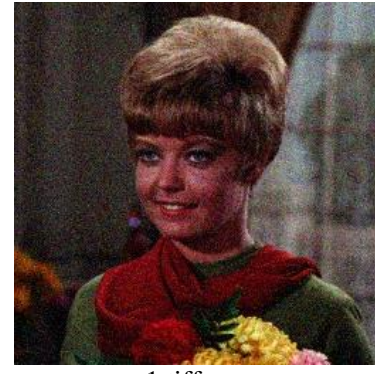

1.tiff

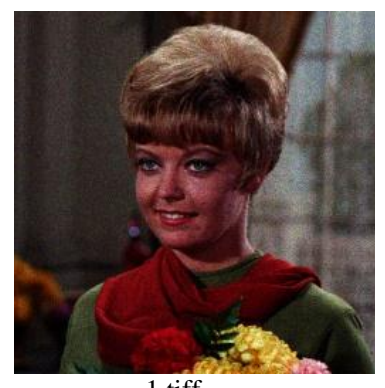

1.tiff

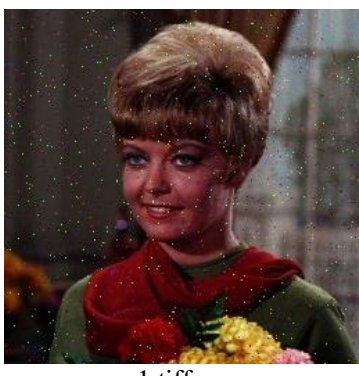

1.tiff

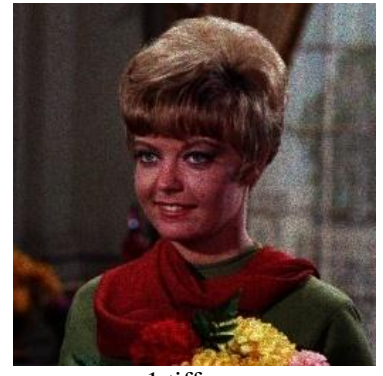

1.tiff

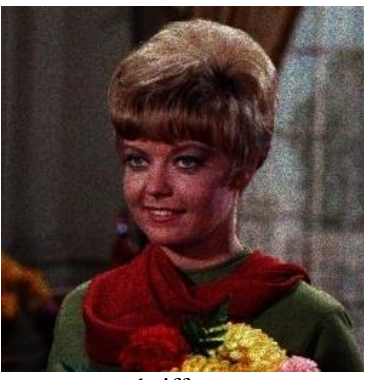

1.tiff

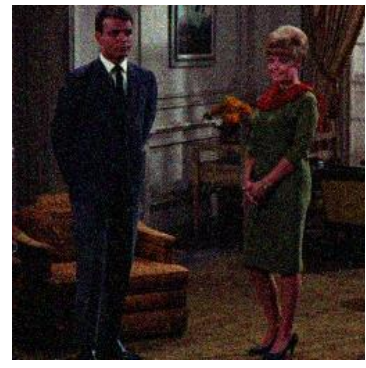

2.tiff

Fig.10. Noisy pictures of Lv noise with 0.01 times of random noise

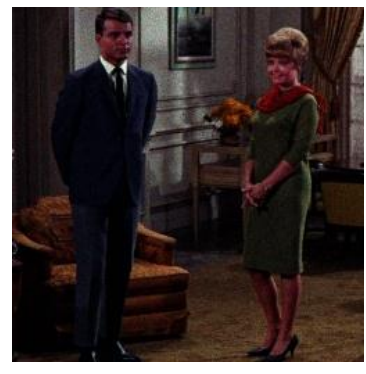

2.tiff

Fig.11. Noisy pictures of Po noise

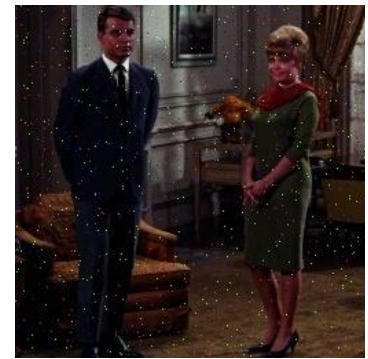

2.tiff

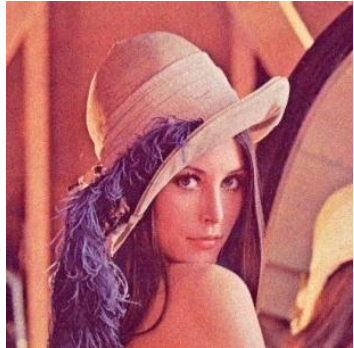

3.tiff

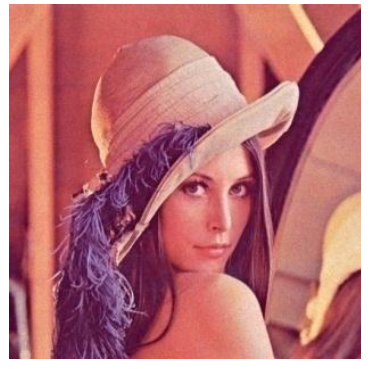

3.tiff

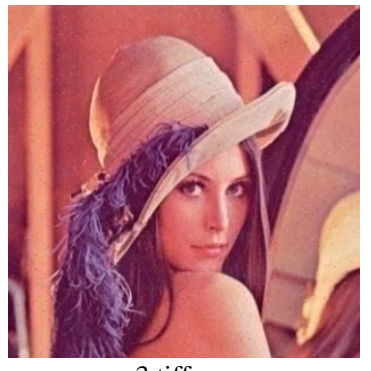

3.tiff
Fig.12. Noisy pictures of S\&P noise with 0.01 density

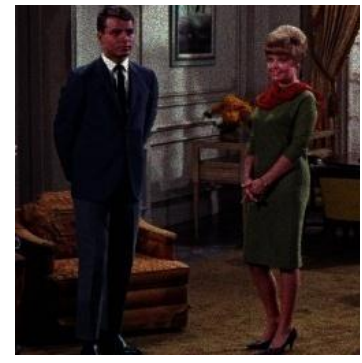

2.tiff

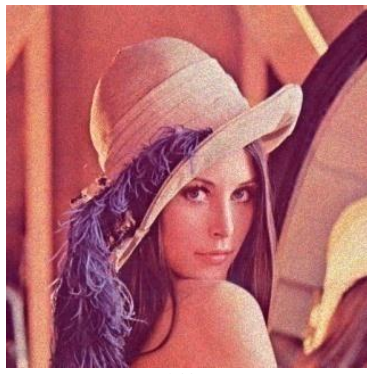

3.tiff

Fig.13. Noisy pictures of Sp noise with 0.02 density

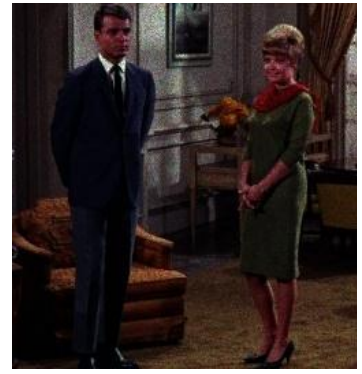

2.tiff

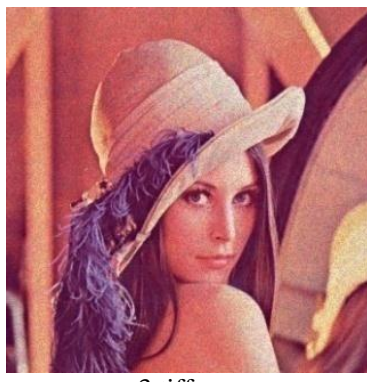

3.tiff

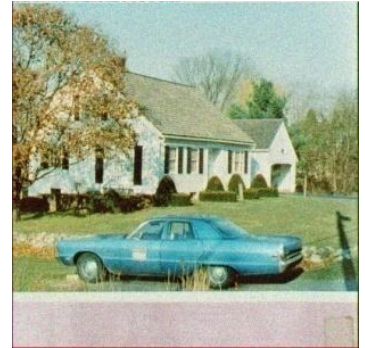

4.tiff

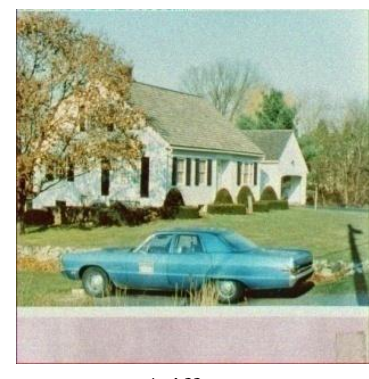

4.tiff
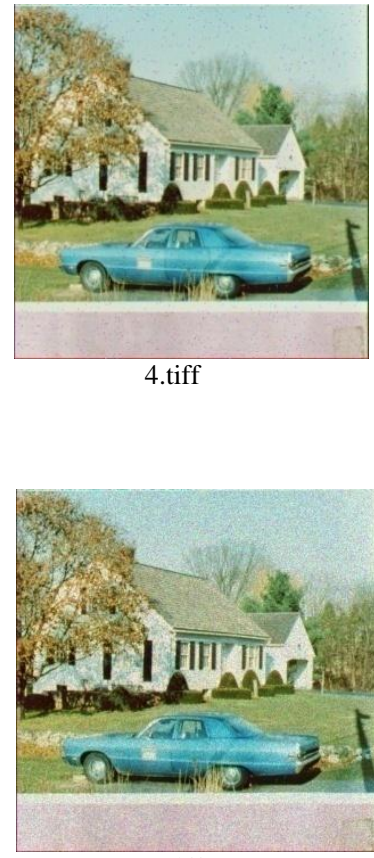

4.tiff

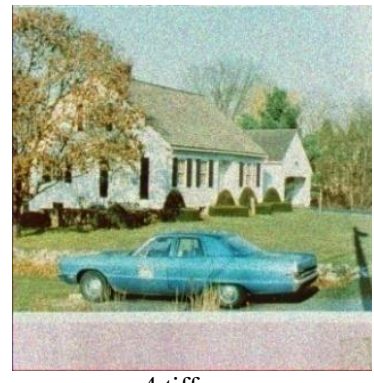

4.tiff

Fig.14. Noisy pictures of Sp noise with 0.03 density 


\section{CONCLUSION}

PQA is a vital issue in the field of DP /VF processing. It is still not satisfyingly comprehended and new methodologies are yet showing up. Our research work focuses on data content weighing with subjective versus objective PQA on NaP. The most utilized measures for PQA in objective and subjective domain are depicted and tested on NaP. The tests in objective domain RMSE, MSE and PSNR with subjective domain SSIM along with content weighing through EnT is demonstrated in which none of the tried strategies can be used alone for PQA functions admirably. The PQA decision should be based on combination of higher SSIM and PSNR with lower MSE and RMSE along with measurable EnT for better PQA.

\section{REFERENCES}

[1] Ajay Kumar Boyat and Brijendra Kumar Joshi, "A Review Paper: Noise Models in Digital Image Processing," Signal \& Image Processing: An International Journal (SIPIJ) Vol.6, No.2, pp. 63-75, 2015.

[2] Nikolay Ponomarenko, Vladimir Lukin, Mikhail Zriakhov,Karen Egiazarian, and Jaakko Astola, "Lossy Compression of Images with Additive Noise," 7th International Conference, ACIVS 2005, Antwerp, Belgium, September 20-23, pp. 381-386, 2005, DOI: 10.1007/11558484_48

[3] David S. Lalush, "Binary Encoding of Multiplexed Images in Mixed Noise," IEEE Transactions on Medical Imaging, vol. 27, no. 9, pp. 1323-1332, 2008, DOI: 10.1109/TMI.2008.922697.

[4] Claude E. Shannon, "Communication in the Presence of Noise," Proceedings of the IEEE, vol. 86, no. 2, February 1998.

[5] Aria Nosratinia, "Post-Processing of JPEG-2000 Images to Remove Compression Artifacts," IEEE Signal Processing Letters,vol. XX, pp. 1-1, 2002.

[6] R. Ramani,N.Suthanthira Vanitha,S. Valarmathy,"The PreProcessing Techniques for Breast Cancer Detection in Mammography Images", International Journal of Image, Graphics and Signal Processing(IJIGSP), vol.5, no.5, pp.47-54, 2013.DOI: 10.5815/ijigsp.2013.05.06.

[7] Yubing Wang, "Survey of Objective Video Quality Measurements," The Institute for Telecommunication Science, pp. 1-7.

[8] Stefan Winkler and Praveen Mohandas, "The Evolution of Video Quality Measurement: From PSNR to Hybrid Metrics The Evolution of Video Quality Measurement: From PSNR to Hybrid Metrics," IEEE transactions on Broadcasting, vol. 54, no. 3, September 2008

[9] M. Kudelka, "Image Quality Assessment," WDS'12 Proceedings of Contributed Papers, Part I, 94-99, ISBN 978-80-7378-224-5, 2012,

[10] Hasan Demirel and Gholamreza Anbarjafari, "Satellite Image Resolution Enhancement Using Complex Wavelet Transform," IEEE geoscience and remote sensing letters, vol. 7, no. 1, pp. 123-126, 2010.

[11] Jincy.C and Rini.M.E, "Performance Analysis of Target Recognition in Synthetic Aperture Radar Images," International Journal of Advanced Research in Electrical, Electronics and Instrumentation Engineering, Vol. 5, Special Issue 2, pp. 171-175, 2016.
[12] S. Sakamoto and H. Mitsuoka, "Totally Mechanized Construction System for High - Rise Buildings (T-UP System)," Proceedings of the 11th ISARC, Brighton, United Kingdom, no. ii, pp. 465-472, 1994.

[13] "Add noise to image - MATLAB imnoise - MathWorks India." [Online]. Available: http://in.mathworks.com/help/images/ref/imnoise.html.

[14] R. Gonzalez and R. Woods, "Digital image processing," Prentice Hall, 2002.

[15] Samuel W. Hasinoff, "Photon, Poisson Noise," Computer Vision. A Reference Guide, Springer Science Business Media New York, pp. 608-610, 2014, DOI:10.1007/9780-387-31439-6_482.

[16] R. H. Chan, C. W. Ho, and M. Nikolova, "Salt-and-pepper noise removal by median-type noise detectors and detailpreserving regularization," IEEE Transactions on Image Processing, vol. 14, no. 10, pp. 1479-1485, 2005.

[17] Jyoti Jaybhay and Rajveer Shastri, "a Study of Speckle Noise Reduction Filters," Signal \& Image Processing : An International Journal (SIPIJ), vol. 6, no. 3, pp. 71-80, 2015, DOI : $10.5121 /$ sipij.2015.6306.

[18] Zhou Wang, Alan C. Bovik, Hamid R. Sheikh and Eero P. Simoncelli, "Image Quality Assessment: From Error Visibility to Structural Similarity," IEEE transactions on image processing, vol. 13, no. 4, pp. 1-14, April 2004.

[19] Je-Ho Park and Changwon Kang, "Entropy Based Image Identifier Generation," in 2014 International Conference on IT Convergence and Security (ICITCS), 2014, pp. 1-2, DOI: 10.1109/ICITCS.2014.7021766.

[20] "Entropy of grayscale image - MATLAB entropy MathWorks India." [Online]. Available: http://in.mathworks.com/help/images/ref/entropy.html.

[21] Suresha D, Prakash H N,"Natural Image Super Resolution through Modified Adaptive Bilinear Interpolation Combined with Contra Harmonic Mean and Adaptive Median Filter", International Journal of Image, Graphics and Signal Processing(IJIGSP), Vol.8, No.2, pp.1-8, 2016.DOI: $10.5815 /$ ijigsp.2016.02.01

[22] G.Rohith and A. Vasuki, "A Novel Approach to Super Resolution Image Reconstruction Algorithm from Low Resolution Panchromatic Images," in 3rd International Conference on Signal Processing, Communication and Networking(ICSCN), 2015, no. x.

[23] Hasan Demirel and Gholamreza Anbarjafari, "IMAGE resolution enhancement by using discrete and stationary wavelet decomposition.," IEEE Transactions on Image Processing, vol. 20, no. 5, pp. 1458-1460, 2011.

[24] "SIPI Image Database - Misc." [Online]. Available: http://sipi.usc.edu/database/database.php?volume=misc. [Accessed: 25-Feb-2016].

\section{Authors' Profiles}

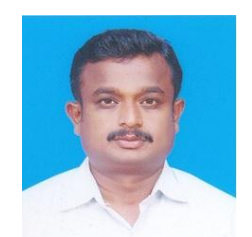

Suresha D is currently a Associate Professor in the Department of Computer Science and Engineering at Canara Engineering College, Mangaluru, India. He received the B.E. \& M.Tech degree in Computer Science \& Engineering from Kuvempu University, Shankaragatta, and Visvesvaraya Technological University, Belagavi, India in 2001 \& 2010 respectively. He is currently a research scholar pursuing $\mathrm{PhD}$ from Visvesvaraya Technological University, Belagavi, India. He has authored several peer reviewed national and international conferences \& Journal papers and his research 
interest includes Image Processing, Computer Vision and Artificial Intelligence.

Prof. Suresha is a life member of professional bodies Computer Society of India, Indian Society for technical education.

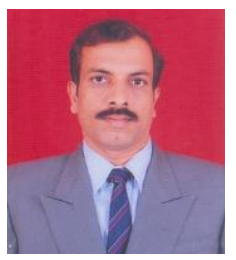

H.N.Prakash received the B.E. degree in Electronics and Communication Engineering from the University of Mysore, Mysore, India, in 1990 and the M.Tech degree in Electronic Instrumentation from Regional Engineering College, Warangal, India in 1995 respectively. He received the
$\mathrm{Ph} . \mathrm{D}$. degree in Computer Science and Engineering from the University of Mysore, Mysore, India in 2010.

$\mathrm{He}$ is currently a Professor in the Department of Computer Science and Engineering at Rajeev Institute of Technology, Hassan, India. He has authored several peer reviewed papers at national and international conferences and Journals Including IEEE Transactions. His research interest includes signature analysis and retrieval, clustering, biometrics, Image processing, Pattern recognition and symbolic data analysis.

Dr. Prakash is a life member of Indian professional bodies such as Institute of Engineers, Indian Society for Technical Education (ISTE) and System Society of India (SSI) and Member of Indian Institute of Engineers, India.

How to cite this paper: Suresha D, H N Prakash,"Data Content Weighing for Subjective versus Objective Picture Quality Assessment of Natural Pictures", International Journal of Image, Graphics and Signal Processing(IJIGSP), Vol.9, No.2, pp.27-36, 2017.DOI: 10.5815/ijigsp.2017.02.04 